\title{
New Strategies on Genetic Engineering and Genome Editing Technologies
}

\author{
Bijoya Bhattacharjee ${ }^{1}$, Lotan Kumar Bose ${ }^{2}$, \\ Nitiprasad Namdeorao Jambhulkar ${ }^{2}$ and Debabrata Nayak ${ }^{3 *}$ \\ ${ }^{1}$ ICAR Research Complex for NEH Region, Umroi Road, Umiam, Meghalaya, India \\ ${ }^{2}$ ICAR-National Rice Research Institute, Cuttack-753006, Odisha, India \\ ${ }^{3}$ Derabis College, Kendrapada, Odisha, India
}

*Corresponding author

\section{A B S T R A C T}

Keywords

Genome editing,

Genetic

engineering, Allele

mining

\section{Article Info}

Accepted:

07 January 2021

Available Online:

10 February 2021
Development of new strategies has always been one of the most important perspectives for molecular biologist. During the last decade, genome editing has received a lot of attention from various researchers across the world. In classical genetics, the gene-modifying activities were carried out selecting genetic sites related to the breeder's goal. Genome engineering is the field in which the sequence of genomic DNA is designed and modified. Genome editing is a technique for genome engineering that incorporate site-specific modifications into genomic DNA using DNA repair mechanisms. Genomics provides breeders with a new set of tools and techniques that allow the study of the whole genome, and which represents a paradigm shift, by facilitating the direct study of the genotype and its relationship with the phenotype. The present review provides information on basic principles, advantages and disadvantages of genome editing technology. The combining of genome research studies with traditional breeding method is essential for crop improvement method. Gene editing differs from genome editing by dealing with only one gene.

\section{Introduction}

\section{Genetic engineering}

Genetic engineering is one of the modern agricultural biotechnology tools that are based on recombinant DNA technology. The term genetic engineering, often interchanged with terms such as gene technology, genetic modification, or gene manipulation, is used to describe the process by which the genetic makeup of an organism can be altered using "recombinant DNA technology." This involves using laboratory tools and specific enzymes to cut out, insert, and alter pieces of DNA that contain one or more genes of interest. The ability to manipulate individual genes and to transfer genes between species that would not readily interbreed is what distinguishes genetic engineering from traditional plant breeding. The process of genetic engineering requires the successful completion of a series of six steps: 
Step 1. Nucleic acid (DNA/RNA) Extraction

Nucleic acid extraction, either DNA or ribonucleic acid (RNA) is the first step in the genetic engineering process. It is therefore important that reliable methods are available for isolating these components from the cell.

\section{Step 2. Gene cloning}

The second step is gene cloning. There are basically four stages in any cloning experiment: generation of DNA fragments, joining to a vector, propagation in a host cell, and selection of the required sequence.

\section{Step 3. Gene Design and Packaging}

Once the gene of interest has been cloned, it has to be linked to pieces of DNA that will control its expression inside the plant cell. These pieces of DNA will switch on (promoter) and off (terminator) the expression of the gene inserted. Gene designing/packaging can be done by replacing an existing promoter with a new one, incorporating a selectable marker gene and reporter gene, adding gene enhancer fragments, introns, and organelle-localizing sequences, among others.

\section{Step 4. Transformation}

The most common methods used to introduce the gene package into the plant cells in a process called transformation or gene insertion, include biolistic transformation using the gene gun and Agrobacteriummediated transformation.

\section{Particle bombardment}

Particle bombardment is a mechanical method of introducing the desired gene. The desired genetic sequence is cloned into a plant DNA vector and introduced into the plant using the gene gun or particle gun.
Agrobacterium tumefaciencs- mediated transformation

The "sharing" of DNA among living forms is well documented as a natural phenomenon. For example, Agrobacterium tumefaciencs, a soil bacterium known as 'nature's own genetic engineer', has the natural ability to genetically engineer plants. It causes crown gall disease in a wide range of broad-leaved plants. Agrobacterium genetically transforms its host by transferring a well-defined DNA segment from its tumor-inducing (Ti) plasmid to the host-cell genome (Gelvin, 1998). Molecular biologists have utilized this biological mechanism to improve crops. The genes that cause the galls are removed and replaced with genes coding for desirable traits. Plant cells infected with the bacterium will not form galls but produce cells containing the desired gene, which when cultured in a special medium will regenerate into plants and manifest the desired trait. The main goal in any transformation procedure is to introduce the gene of interest into the nucleus of the cell without affecting the cell's ability to survive. If the introduced gene is functional, and the gene product is synthesized, then the plant is said to be transformed. Once the inserted gene is stable, inherited and expressed in subsequent generations, then the plant is considered a transgenic.

The molecular machinery needed for T-DNA production and transport into the host cell comprises of proteins that are encoded by a set of bacterial chromosomal (chv) and $\mathrm{Ti}$ plasmid virulence (vir) genes. In addition, various host proteins have been reported to participate in the Agrobacterium- mediated genetic transformation process (Gelvin, 2003; Txfira and Citovsky, 2006), mostly during the later stages of the process (i.e. T-DNA intracellular transport, nuclear import and integration). The vir region, located on the 
Agrobacterium Ti plasmid, encodes most of the bacterial virulence (Vir) proteins used by the bacterium to produce its T-DNA and to deliver it into the plant cell Tzfira and Citovsky (2002). Vir gene is composed of at least seven major loci (virA, virB, virC, virD, virE, virF, and virG). Virulence gene expression leads to the production of a singlestranded T-DNA, termed the T-strand, which is then transported into the host cell. T-DNA integration into the plant cell genome is the final step of the Agrobacterium-mediated transformation process.

The major steps involve in the Agrobacterium tumefaciencs-mediated plant transformation process includes the following procedures (Hwang et al., 2017).

(1) Attachment of A. tumefaciens to the plant cells.

(2) Sensing plant signals by A. tumefaciens and regulation of virulence genes in bacteria following transduction of the sensed signals.

(3) Generation and transport of T-DNA and virulence proteins from the bacterial cells into plant cells.

(4) Nuclear import of T-DNA and effector proteins in the plant cells.

(5) T-DNA integration and expression in the plant genome.

The A. tumefaciens-mediated transformation method has several advantages over other transformation methods; it is easy to use, relatively inexpensive, and generally results in low copy number and well-defined DNA insertions into the host cell chromosome (Koncz et al., 1994; Pawlowski and Somers, 1996; Hansen et al., 1997; Enríquez-Obregón et al., 1998; Shou et al., 2004; Travella et al., 2005; Zhang et al., 2005; Gao et al., 2008).
Agrobacterium also offers a number of advantages over Biolistic-mediated techniques in terms of efficiency and the quality of the transformed plants produced (Harwood et al., 2009).

Most of the progress achieved to date in establishing protocols for the transformation of new host species has relied on a relatively small number of binary vectors and genetically modified Ti-helper plasmids, and on an even smaller number of disarmed Agrobacterium strains and isolates. Thus, progress in the genetic transformation of different plant species has been mostly achieved by matching the inoculated plant tissue to the suitable Agrobacterium strain, by genetic modification of Agrobacterium, and by developments in tissue culture and transgene selection techniques (Estrella et al., 2005).

Recent studies of Agrobacterium-host interactions that focus on revealing the functions of host proteins in the transformation process (Tzfira and Citovsky, 2006; Gelvin, 2003) hold great potential for the future of the biotechnology of plant genetic engineering.

\section{Gene stacking}

Gene stacking strategy that may be used by Agrobacterium-based delivery of the integrating DNA is a transformation operating system that will help shorten the development time of transgenic plants as well as help mitigate biosafety concerns of plant genetic modifications.

\section{Step 5. Detection of inserted genes}

Molecular detection methods have been developed to determine the integrity of the transgene (introduced gene) into the plant cell. 


\section{Polymerase chain reaction $(\mathrm{PCR})$}

PCR is a quick test to determine if the regenerated transgenic cells or plants contain the gene.

\section{Southern blot analysis}

Southern blot analysis determines the integrity of the inserted gene: whether the gene is complete and not fragmented, at the correct orientation, and with one copy number.

\section{Northern blot analysis}

Northern blot analysis determines whether the transcript or the messenger RNA (mRNA) of the introduced DNA is present and is correctly transcribed in the transgenic plant.

\section{Western blot analysis}

Western blot analysis or protein immuneblotting is an analytical technique used to detect whether the transgenic plants produce the specific protein product of the introduced gene

\section{Step6 Field trial}

Genetic transformation is usually conducted in elite or commercial varieties which already possess the desired agronomic traits but lacks the important trait of the transgene. Thus, once successfully conducted, the genetically modified plant will be easily recommended for commercialization if it shows stability in several generations and upon successfully passing and fulfilling varietal registration requirements.

\section{Case study of transgenic crops}

Transgenic crops are now grown commercially on several million hectares, the predominant crops being maize, soybean, cotton and soybean (Dunwell, 2000). Genetically-modified (GM) crops proved to be powerful complements to those produced by conventional methods for meeting the worldwide demand for quality foods. GM crops enhance yields, nutritional quality and increase tolerance to various biotic and abiotic stresses. Genetic engineering and plant transformation have played a pivotal role in crop improvement via introducing beneficial foreign gene(s) or silencing the expression of endogenous gene (s) in crop plants. Genetic engineering technology allows the transfer of a single gene, or a couple of genes, in a much more precise, controllable and predictable way than is achievable with conventional breeding.

Expressions of concerns about biosafety and health hazards associated with GM crops, has been debated worldwide although there is no reason to hesitate in consuming geneticallyengineered food crops that have been thoughtfully developed and carefully tested. Modern biotechnology integrated with conventional agricultural practices in a sustainable manner, can fulfil the goal of attaining food security for present as well as future generations Datta (2013). Backcrossing, a traditional approach for bringing the desirable gene to the progeny is a time consuming method and sometimes bring along some undesirable gene which is unsafe. For example, in 1986, toxic levels of the glycoalkaloids $\alpha$-solanine and $\alpha$ - chaconine were found in potato variety magnum bonum. These glycoalkoloids cause alkaloid poisoning leading to gastrointestinal, circulatory, neurological and dermatological problems. All somatic hybrids of $S$. tuberosum and $S$. brevidensproduce a toxin demissidine in the leaves, which is not produced in either of the parents. The technology of genetic engineering employs a precise control on the timing and location of 
gene products resulting in tissue/organ/development/stress-specific expression - an outcome not easy to accomplish with classical breeding.

\section{Genomics assisted breeding technology}

Genomics provides breeders with a new set of tools and techniques that allow the study of the whole genome, and which represents a paradigm shift, by facilitating the direct study of the genotype and its relationship with the phenotype (Tester and Langridge, 2010). Functional markers and informatics have been laid by advanced genomics research as well as new knowledge about statistics and inheritance phenomena which could increase the efficiency and precision of crop improvement (Varshney et al., 2005).

The combining of genome research studies with traditional breeding method is essential for crop improvement method. A fully sequenced and well annotated genome provides useful tools for the breeders, as it allows the discovery of genes, determining their position and function, as well as the development of large marker collections and high resolution maps (de-Castro et al., 2012).

Advances in genomics can contribute to crop improvement in two general ways. First, a better understanding of the biological mechanisms can lead to new or improved screening methods for selecting superior genotypes more efficiently. Second, new knowledge can improve the decision-making process for more efficient breeding strategies. Genomics approaches are particularly useful when dealing with complex traits, as these traits usually have a multi-genic nature and an important environmental influence. The work of a genome assembly is a complex task requiring powerful computers and skilled bioinformatics.
There are three classes of genomic approach namely functional genomics, structural genomics and comparative genomics.

\section{Functional genomics}

Functional genomics unravel gene functions and the interactions between genes in regulatory networks, which can be exploited to generate improved varieties. Sequencing based approach and hybridization based approach are two ways to explored the gene catalogue of a species. Expressed Sequence Tag (EST) is sequence based approach which has been shown to identify corresponding genes unambiguously in a rapid and costeffective fashion (Bouchez and H“ofte, 1998). Therefore, ESTs have been a major focus on functional studies. cDNA libraries from various tissues, developmental stages, or treatments generally serve as the sources for EST sequencing to reveal differentially expressed genes (Yamamoto and Sasaki, 1997). ESTs are usually much shorter in length than the cDNAs from which they are obtained. EST sequencing is mostly used in the absence of whole genome sequences, particularly in crops with large and repetitive genomes, although the entire transcriptome is unlikely to be fully represented and resolved (Akpinar et al., 2013). Even so, EST sequencing is still a valid approach, and a recent study has demonstrated its potential in gene discovery via the comparison of different genotypes under control and stress conditions (Ergen and Budak, 2009).

Array-based techniques or hybridization techniques utilize hybridization of the target DNA with cDNA or oligonucleotide probes attached to a surface to assess expression. These array-based methods are targeted; that is, prior knowledge of the transcript to be analysed, either sequence or clone, is a prerequisite to design probes. The choice of tissue or genotype that is sampled in a 
microarray study is related to the relevance of results.

\section{Allele mining or Eco tilling}

To know a relative value of all alleles for genes of interest in the primary germplasm a breeder would ideally like to adopt plant breeding strategies for crop improvement. This process is known as 'allele mining'. A strategy based on targeting induced local lesions in genomes (TILLING), was developed for detecting multiple types of polymorphisms in germplasm collections (e.g. natural population, breeding or gene bank materials) (Caldwell et al., 2004). Targeting Induced Local Lesions IN Genomes (TILLING) applicable to virtually all genes in all species where mutations can be induced and has been reported in several crop species, including hexaploid wheat. TILLING populations are conventionally screened by phenotypic or genotypic variations; further use of certain TILLING mutants in elucidation of stress responses has been demonstrated. It takes advantage of classical mutagenesis, sequence availability and high throughput screening for nucleotide polymorphisms in a targeted sequence. TILLING protocols involves the use of bioinformatics tools, new methods of mutation detection, including mismatchspecific and sensitive endonucleases. It also involves screening for single nucleotide polymorphism (SNP) discovery using nextgeneration technologies.

Importantly, a modified strategy, called EcoTILLING, has been developed to identify natural polymorphisms, analogous to TILLING-assisted identification of induced mutations. EcoTILLING allows natural alleles at a locus to be characterized across many germplasm. EcoTILLING is expected to provide a series of alleles for those genes that are involved in important processes of the plant even though the known variants for these genes have not been observed through genetic studies. Extensive information about the candidate genes in terms of structure and regulation or phenotypic expression is important for designing the primer pairs for EcoTILLING. After identifying all alleles that are available, they must be evaluated for their relative value in adapted genotypes in the target environment. These analyses might help in designing synthetic alleles that are superior to those found in nature. This could be accomplished by recombining the coding regions of genes either randomly (e.g. by gene shuffling) or deliberately (e.g. by domain swapping). EcoTILLING is able to implicate favourable haplotypes for further analyses, such as sequencing.

\section{Structural genomics}

Structural genomics focus on the physical structure of the genome, aiming to characterize genome structures. Together, structural genomics and functional genomics can characterize a genome to its full extent. Structural genomics projects aim to solve the experimental structures of all possible protein folds. When applied to genomes, structural information (either experimental or predicted) is likely to play an important role in highthroughput function assignment. Structural genomics aims to first determine the structure of proteins, and then investigate their function later.

\section{Comparative genomics}

Comparative genomics is a promising tool to gain information by utilizing conservation between closely related plant species. Comparative genomics has contributed significantly to the emergence of the "genome zipper" concept, which enables the determination of a virtual gene order in a partially sequenced genome. 


\section{Genome sequencing and mapping}

The Sanger technology has been the predominant sequencing method for the past thirty years. It has been used to sequence several genomes as well as many transcriptomes. With the arrival of NGS the field of genomics has been changed, through NGS technologies, sequencing and resequencing of even large genomes have become feasible. Re-sequencing of genomes is very useful for the genome-wide discovery of markers amenable for high-throughput genotyping platforms, like SSRs and SNPs, or the construction of high density genetic maps. Re-sequencing not only avoids repetitive sequences of complex genome but also identifies SNPs within transcripts that may serve as functional markers. Both Roche 454 and Illumina GA have been mostly used for genome re-sequencing. Based on research findings of de-Castro et al., (2012), they found out that Next Generation Sequencing (NGS) technologies allowed mass sequencing of genomes and transcriptomes, producing a vast array of genomic information. The analysis of NGS data by means of bioinformatics developments allows discovering new genes and regulatory sequences and their positions, and makes available large collections of molecular markers. Genome-wide expression studies provide breeders with an understanding of the molecular basis of complex traits. NGS also provide plant breeders the information of genetic variation present in the genome. NGS have reduced the cost of sequencing by more than one thousand times compared to Sanger technology, by avoiding time consuming and tedious traditional cloning steps and making possible to perform millions of sequencing reactions. The second and third generation sequencing has also been developed but only the second generation sequencing has been used profusely for crop sequencing.

\section{Genome editing}

Genome editing involves the usage of engineered site-specific nucleases (SSNs) to modify specific genes at desired locations in the genome. It involves insertion, deletion, or replacement of a segment of DNA in the genome of an organism. The SSNs such as zinc finger nucleases (ZFNs), transcriptional activator-like effector nucleases (TALENs) and clustered regularly interspaced short palindromic repeats (CRISPR)-associated endonuclease Cas9 (CRISPR/Cas9) make a double-stranded break (DSB) in the target DNA which is subsequently repaired by cell's own natural repair mechanism of homologous recombination (HR) or non-homologous end joining (NHEJ) (Miglani, 2017). The most advanced genome editing tool in plant biology is clustered regularly interspaced short palindromic repeats associated endonuclease Cas9 (Belhaj et al., 2015; Weeks et al., 2016). It consists of a short RNA molecule called guide RNA which is associated with a DNA endonuclease called Cas9. CRISPR-associated protein 9 (Cas9) is a DNA endonuclease responsible for cutting the invading phage DNA into pieces, which then gets integrated into the CRISPR array as a spacer. The guide RNA is a two-component system consisting of the crRNA (CRISPRderived RNA) and tracr RNA (transactivating RNA). In nature, the crRNA targets the double stranded DNA to be cut, and has a short region of homology allowing it to bind the tracr RNA. The tracr RNA provides a stem loop structure which associates with Cas9 protein. In the CRISPR/Cas9-based genome editing system, the crRNA and tracrRNA were engineered into a single guide RNA chimera (sgRNA) that can also direct sequence-specific Cas9 dsDNA cleavage (Jinek et al., 2012). The protein/RNA complex (Cas9-sgRNA) moves along the DNA strand and makes a double stranded break (DSB) where the sgRNA matches the 
target DNA sequence (Jinek et al., 2014). The application of genome editing tools has broadened rice research, bringing in new opportunities to develop novel varieties with improved productivity and quality (Mishra et al., 2018). CRISPR/cas9 enables to develop mutation at desired targets in the genome with greater accuracy, efficiency, and simplicity thereby, creating variation in the population of crop species which is an important tool in plant breeding.

To edit a plant genome, the CRISPR-Cas9 construct has to be transformed into plant cells, and whole plants have to be regenerated from the few transformed cells. Genome editing has mostly been applied in transformable plants. Current transformation methods are normally genotype specific, and transformation procedures remain to be established for many plant species. Moreover, Agrobacterium, used for transforming most plants, raises regulatory concerns as it is considered a plant pathogen. Therefore, plant transformation is a major bottleneck for realizing the potential of plant genome editing. Experimental methods addressing DNA delivery and plant transformation issues deserve more intensive investigation.

In conclusion through this review, we have come across many new strategies in plant molecular biology such as tissue culture techniques, marker assisted selection using DNA markers, genetic engineering technology, genome sequencing and genome editing. The principle and steps of every techniques used here are discussed here. The impact of tissue culture such as embryo culture has been extensively used in distant wide hybridization in overcoming the postzygotic failure. Embryo culture in vitro is also used to overcome dormancy of recalcitrant seeds. Plant breeder have also been extensively used the techniques of haploid plant production in order to get homozygous plants thereby, shortening the breeding cycle. After undergoing chromosome doubling the chromosome set of a haploid plant has been doubled spontaneously and become fertile. Micro propagation was mainly used in mass production of flowering plants, conserving the germplasm of flowers, production of synseedsin orchid through the encapsulation of PLBs as the survival rate is high after grown in greenhouse for six months. The use of molecular markers in plant breeding has been of great help to plant breeders in indirect selection processes, enabling manual selection of individuals with a particular trait of interest. Markers assisted selection also is of great help in backcrossing process. A thorough selection for parents which are diverse containing desired traits and developing a mapping population for screening the trait of interest are important in marker assisted selection. The constraint of using Marker Assisted Selection is that not all markers can be applicable across populations due to lack of marker polymorphism or reliable marker-trait association. Technical skill is required starting from the selection of parents, developing mapping population handling of equipment, isolation of DNA molecules and study of DNA markers.

Genetic engineering and plant transformation have played a pivotal role in crop improvement via introducing beneficial foreign gene(s) or silencing the expression of endogenous gene (s) in crop plants. Genetic engineering technology has overcome conventional breeding in a predictable way by allowing the transfer of a single gene, or a couple of genes. Transgenic crops have also been developed in many crops the predominant crops being maize, soybean, cotton and soybean. Transgenic crops enhance yields, nutritional quality and increase tolerance to various biotic and abiotic stresses. 
Advances in genomics have contributed to crop improvement allowing the study of the whole genome, facilitating the direct study of the genotype and its relationship with the phenotype improved screening methods for selecting superior genotypes more efficiently. Genomics approaches are particularly useful when dealing with complex traits, as these traits usually have a multi-genic nature and an important environmental influence. Genomics required powerful computers and skilled bioinformatics which are the main constraint and challenges faced by plant breeders.

Genome editing technologies allows modifying specific genes at desired locations in the genome which involves insertion, deletion, or replacement of a segment of DNA in the genome of an organism. New breeding technologies based on genome editing that allow simultaneous modification of multiple genetic loci in elite varieties will accelerate crop improvement and enhance global food security. However, information on genome sequences and gene functions is a precondition for effective genome editing.

Plant genome editing still faces challenges, primarily in terms of establishing a unified delivery method and manipulation of DNA repair pathways. CRISPR-based genome editing has been quickly established as a powerful tool that is revolutionizing plant biology research and crop trait development, in a manner similar to that brought about by molecular cloning and PCR technologies.

In a future perspective it is clearly indicated that these technologies developed by biotechnologist are of great help in crop improvement. It shortened breeding cycle and overcome the difficulties of conventional method of plant breeding. New hybrids and improved cultivars can be developed in a short span of time using these technologies.

\section{References}

Akpinar, B. A., S. J. Lucas, and Budak, H. 2013. Genomics approaches for crop improvement against abiotic stress. Sci. World J. 36, 19-21. DOI: $10.1155 / 2013 / 361921$

Belhaj, K., A. Chaparro-Garcia, S. Kamoun, N. J. Patron, and Nekrasov, V. 2015. Editing plant genomes with CRISPR/Cas9. Curr. Opin. Biotechnol. 32, 76-84. DOI: 10.1016/j.copbio.2014.11.007

Bouchez, D., and H“ofte, H. 1998. Functional genomics in plants. Plant Physiology. $118,725-732$.

Caldwell, D.G., N. McCallum, P. Shaw, G. J. Muehlbauer, D. F. Marshall, and Waugh, R. A. 2004. Structured mutant population for forward and reverse genetics in Barley (Hordeum vulgare L.). Plant J. 40, 143-150.

Datta, A., 2013. Genetic engineering for improving quality and productivity of crops. Agriculture \& Food Security. 2, 15.

De-Castro, A.M.P., Vilanova, S., Cañizares, J., Pascual, L., Blanca, J.M., Díez, M.J., Prohens, J. and Picó.B. 2012. Application of Genomic Tools in Plant Breeding. Current Genomics. 13, 179195

Dunwell, J.M. 2000. Transgenic approaches to crop improvement. Journal of Experimental Botany. 51 (1), 487-496.

Enríquez-Obregón, G. A., R. I. Vázquezpadrón, D. L. Prieto-sansonov, G. A. de la Riva, and Selman-Housein, G. 1998. Herbicide resistant sugarcane (Saccharum officinarum L.) plants by Agrobacterium-mediated transformation. Planta 206, 20-27.

Ergen, N.Z., and Budak, H. 2009. Sequencing over 13000 expressed sequence tags from six subtractive cDNA libraries of wild and modern wheats following slow 
drought stress. Plant, Cell and Environment. 32 (3), 220-236.

Estrella L. H., S. June, and Martinez-Trujillo Miquel. 2005. Transgenic plants: An Historical Perspective. Methods in Molecular Biology. 286, 3-32.

Gao, C., D. Long, I. Lenk, and Nielsen, K. K. 2008. Comparative analysis of transgenic tall fescue (Festuca arundinacea Schreb.) plants obtained by Agrobacterium-mediated transformation and particle bombardment. Plant Cell Rep. 27, 1601-1609.

Gelvin S. B. (1998). Agrobacterium VirE2 proteins can form a complex with $\mathrm{T}$ strands in the plant cytoplasm. J. Bacteriol. 180, 4300-4302.

Gelvin, S.B. 2003. Agrobacterium-mediated plant transformation: the biology behind the 'gene-jockeying' tool. Microbiology and Molecular Biology Review. 67, 1637.

Hansen G., R. D. Shillito, and Chilton M.D. 1997. T-strand integration in maize protoplasts after codelivery of a T-DNA substrate and virulence genes. Proc. Natl. Acad. Sci. USA. 94(21). 11726 11730

Harwood, W. A., Bartlett, J., Alves, S., Perry, M., Smedley, M., Leyland, N., Snape, J.W. 2009. Barley transformation using Agrobacterium-mediated techniques. In: Methods in Molecular Biology Transgenic Wheat, Barley and Oats (Eds.) Huw D Jones and Peter R Shewry, Pp. 137-147.

Hwang, H. H., M. Yu, and Lai, E. M. 2017. Agrobacterium-mediated plant transformation: biology and applications. The Arabidopsis Book, 2017(15): $\quad$ (2017). DOI: https://doi.org/10.1199/tab.0186

Jinek, M., F. Jiang, D. W. Taylor, S. H. Sternberg, E. Kaya, E. Ma, C. Anders, M. Hauer, K. Zhou, S. Lin, M. Kaplan,
A. T. Iavarone, E. Charpentier, E. Nogale and Doudna, J. A. 2014. Structures of Cas9 endonucleases reveal RNA-mediated conformational activation. Science 343, 61-76. DOI: $10.1126 /$ science. 1247997

Jinek, M., K. Chylinski, I. Fonfara, M. Hauer, J. A. Doudna, and Charpentier E. 2012. A programmable dual-RNA-guided DNA endonuclease in adaptive bacterial immunity. Science 337, 816-821.

Koncz, C., N. Martini, L. Szabados, M. Hrouda, A. Bachmair, and Schell, J. 1994. Specialized vectors for gene tagging and expression studies. In: Gelvin, S. B., R. A. Schilperoort, and Verma, D. P. S.(Ed.), Plant Molecular Biology Manual, Ed 2, Vol B. Kluwer Academic Publisher, Dordrecht, The Netherlands, pp 1-22

Miglani, G. S. 2017. Genome editing in crop improvement: Present scenario and future prospects. Journal of Crop Improvement, 31(4), 453-559. DOI: $10.1080 / 15427528.2017 .1333192$

Miglani, G.S. 2017. Genome editing in crop improvement: Present scenario and future prospects. Journal of Crop Improvement 31 (4), 453-559.

Mishra, J., T. Fatima, and Arora, N. K. 2018. Role of secondary metabolites from plant growth-promoting rhizobacteria in combating salinity stress. In: Ahmad P., Egamberdieva D. (Ed.), Plant Microbiome: Stress Response, (Singapore: Springer; ), 127-163. DOI: 10.1007/978-981-10-5514-0_6

Pawlowski, W.P., and Somers D. A. 1996. Transgene inheritance in plants genetically engineered by microprojectile bombardment. Molecular Biotechnology 6, 17-30.

Shou, H., B. R. Frame, S. A. Whitham, and Wang, K. 2004. Assessment of transgenic maize events produced by particle bombardment or Agrobacterium 
mediated transformation. Mol. Breed. 13, 201-208.

Tester, M., and Langridge, P. 2010. Breeding technologies to increase crop production in a changing world. Science. 327,818 822.

Travella, S., S. M. Ross, J. Harden, C. Everett, J. W. Snape, and Harwood W. A. 2005. A comparison of transgenic barley lines produced by particle bombardment and Agrobacterium mediated techniques. Plant Cell Rep. 12, 780-789.

Tzfira, T., and Citovsky, V. 2002. Partners-ininfection: host proteins involved in the transformation of plant cells by Agrobacterium. Trends Cell Biology. 12, 121-129.

Tzfira, T., and Citovsky, V. 2006. Agrobacterium-mediated genetic transformation of plants: Biology and biotechnology. Current Opinion in Biotechnology, 17(2), 147-154. DOI: 10.1016/j.copbio.2006.01.009.

Varshney, R.K., Graner, A., and Sorrells, M.E. 2005. Genomics-assisted breeding for crop improvement. Trends in Plant Science. 10 (12), 621-629.

Weeks, D. P., M. H. Spalding, and Yang, B. 2016. Use of designer nucleases or target gene and genome editing in plants. Plant Biotechnology J. 14, 483495.

Yamamoto, K., and Sasaki, T. 1997. Largescale EST sequencing. Plant Mol. Biol. 35, 135-144.

Zhang, Y., X. Yin, A. Yang, G. Li, and Zhang J. 2005. Stability of inheritance of transgenes in maize (Zea mays L.) lines produced using different transformation methods. Euphytica 144, 11-22.

\section{How to cite this article:}

Bijoya Bhattacharjee, Lotan Kumar Bose, Nitiprasad Namdeorao Jambhulkar and Debabrata Nayak. 2021. New Strategies on Genetic Engineering and Genome Editing Technologies. Int.J.Curr.Microbiol.App.Sci. 10(02): 662-672. doi: https://doi.org/10.20546/ijcmas.2021.1002.080 